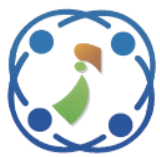

\title{
Identification of Diabetes through Urine Using Gas Sensor and Convolutional Neural Network
}

\author{
Misbah $^{1} \quad$ Muhammad Rivai $^{1 *} \quad$ Fredy Kurniawan $^{2} \quad$ Zainul Muchidin $^{3} \quad$ Dava Aulia $^{4}$ \\ ${ }^{1}$ Department of Electrical Engineering, Institut Teknologi Sepuluh Nopember Surabaya, Indonesia \\ ${ }^{2}$ Department of Chemistry Engineering, Institut Teknologi Sepuluh Nopember Surabaya, Indonesia \\ ${ }^{3}$ Laboratorium Klinik, Rumah Sakit Petrokimia Gresik, Indonesia \\ ${ }^{4}$ Department of Computer Engineering, Institut Teknologi Sepuluh Nopember Surabaya, Indonesia \\ *Corresponding author's Email: muhammad_rivai@ee.its.ac.id
}

\begin{abstract}
Currently, blood glucose disease detection devices are administered invasively by inserting a needle into a finger or a blood vessel. This can cause trauma to the patient, especially if the frequency of examinations is very frequent. Therefore, we need a more comfortable and effective device for early recognition of the signs of diabetes mellitus. Blood sugar monitoring can also be done by smelling the patient's urine. Electronic nose is a system that can be used to identify someone with diseases including diabetes. The electronic nose system used in this study consists of several semiconductor gas sensors. The pattern recognition algorithms utilize convolutional neural network (CNN), learning vector quantization (LVQ), multilayer Perceptron (MLP), Naïve Bayes, k-Nearest Neighbors (k-NN), SVM, PCA-Logistic Regression (PCA-LR), PCA -LVQ, and PCA-MLP. The experimental results show that the CNN has the highest accuracy of $100 \%$ in the identification of two classes, namely healthy, and diabetic.
\end{abstract}

Keywords: Convolutional neural network, Diabetes, Diseases, Gas sensor, Urine.

\section{Introduction}

Diabetes is a dangerous chronic disease. This disease can cause various complications, such as stroke [1], cardiovascular disease [2], eye disorders [3], kidney disease [4], and neurological disorders [5]. This disease is characterized by increased levels of sugar (glucose) in the blood. Insulin, which is secreted from cells, is distributed evenly throughout the pancreas to lower glucose. Lack of insulin or insulin resistance directly causes high blood glucose during fasting and after eating [6]. High blood glucose levels increase the amount of glucose filtered by the kidneys. If the filtered glucose exceeds the amount that can be reabsorbed by the kidneys, the excess glucose will enter the urine [7]. In diabetics, the levels of volatile organic compounds (VOC) in the urine will change. Substances that have changed include: succinic acid, L-leucine, L-isoleucine, tyrosine, acetoacetic acid, mannose, L-isoleucine, L- threonine, Phenylalanine, fructose, D-glucose, palmitic acid, oleic acid, and arachidonic acid [8]. So far, clinical diagnosis has been based on checking blood sugar. This diagnosis is considered fast and accurate. However, the possibility of infection and pain during blood sampling can also be a problem. Clinical sampling by detecting glucose in urine is considered safer and cheaper. Electronic nose is a system that can help solve these problems. This method is more effective because this system can detect glucose and volatile organic compounds in the patient's urine.

Electronic nose has been widely used for identification of sweetness of pineapple [9], detection of toxic substances [10], monitoring fish quality [11], detection of civet and non-civet coffee [12], and identification of asthma [13].

The electronic nose consists of several sensors and a data processing unit. In the data processing unit, there is a pattern recognition algorithm that can be used to identify diseases. Various types of sensors 
have been used to detect diabetes, including Localized Surface Plasmon Resonance (LSPR) [14], fluorescence [15], electrodes [16], Quartz Crystal Microbalance (QCM) [10, 17], and metal oxide [18]. LSPR and fluorescence are types of optical sensors that have the disadvantage of being expensive, having complex systems, and less portable. Likewise, sensor electrodes are large in size and have limited sensitivity. The disadvantages of the QCM system are a complex circuit, interference occurs between channels, and sensitive to temperature and humidity. Meanwhile, metal oxide sensors have the advantages of high sensitivity, fast response, low cost, having simple circuit, and portable.

Pattern recognition methods to identify diabetes can be done using cluster analysis (CA) and Principal Component Analysis (PCA) [16], Partial Least Square-Discriminant Analysis (PLS-DA), PCA [8], K-Support Vector Machine (K-SVM) [19], and neural network [20].

The CA, PCA, PLS-DA, and K-SVM methods do not use the learning process so that sometimes the results are not satisfactory. Meanwhile, the neural network uses the learning process to make it better because it can adapt to unknown conditions. Preprocessing of data in neural networks becomes very important in the success of pattern recognition. The averaging method is often used in pre-processing. In addition, there is also a convolution method, which offers better capabilities in pre-processing.

In this study, an electronic nose-based identification system for diabetics and healthy patients is proposed. The sample is taken from the patient's urine vapor, so this method is cheap, easy, comfortable, and avoids infection. Metal oxide sensors used in this system include MQ-2, MQ-4, MQ-7 and MQ-135. The pattern recognition algorithm utilizes the CNN method. This method has the ability to jointly learn features and classification for raw input data. Convolution kernel is a filter that can extract features from raw data [21]. The comparison methods include LVQ, MLP, Naïve Bayes, k-Nearest Neighbors (k-NN), SVM, PCALogistic Regression (PCA-LR), PCA-LVQ, and PCA-MLP. This study contributes to the application of gas sensors to analyze disease through urine vapor, in addition to getting the best CNN configuration in the identification of diabetes.

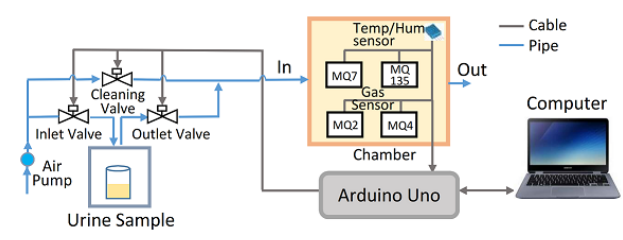

Figure. 1 Electronic nose system design

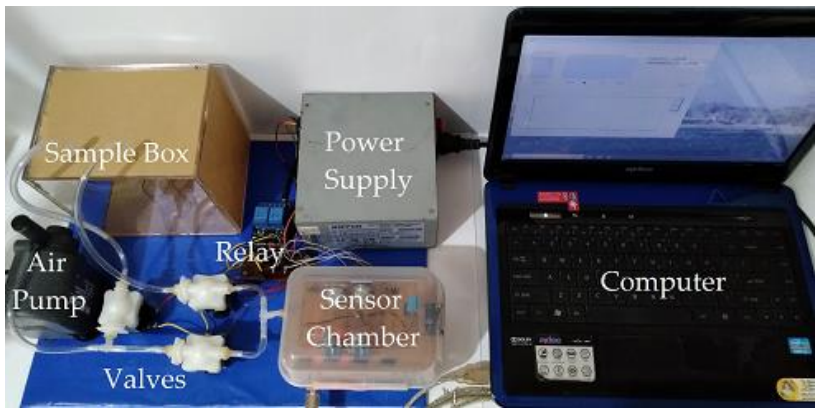

Figure. 2 Experimental equipment

\section{Methods}

The design of the electronic nose system in this study consisted of a metal oxide gas sensors array, an Arduino Uno microcontroller as a data acquisition unit, and a valve control as shown in Fig. 1. The experimental equipment used in this study is shown in Fig. 2. The size of the urine sample box is $1.7 \mathrm{~L}$. The chamber sensor volume is $0.6 \mathrm{~L}$, in which there are 4 gas sensors of MQ2, MQ4, MQ7, MQ135, and a DHT11 sensor to measure temperature between 0 $50^{\circ} \mathrm{C}$ and humidity between $20-90 \%$ RH. All gas sensors are connected to the Arduino Uno module which has 6 analog inputs with 10-bit ADC resolution. The data obtained from the sensor is sent to the computer via USB serial communication. Sensor responses and gas concentrations taken from the patient's urine are stored by the electronic nose in the form of a text file (.txt). For sensor data and gas concentrations in several patients are accompanied by medical diagnoses recorded in the database. Before measuring the urine sample, the chamber is first cleaned by turning on the clean air pump and opening the cleaning valve. After that, the sample is inserted by opening the valve in and valve out, and closing the cleaning valve.

\subsection{Urine sample}

Data were taken from urine specimens of diabetic and healthy patients as many as 50 people. The patients consisted of 20 women and 30 men with an age range of 35-60 years old at Petrokimia Gresik Hospital. There were 50 urine samples used in this study, consisting of 40 samples of diabetics and 10 healthy samples. The urine sample is poured into a sample glass of about $6 \mathrm{ml}$. The temperature and humidity in the chamber are measured between $25^{\circ} \mathrm{C}$ $-30^{\circ} \mathrm{C}$ and $35 \%-45 \% \mathrm{RH}$.

\subsection{Sensor selection}

In previous studies, the identification of diabetes was carried out by sensing six types of volatile organic compounds (VOCs) such as ammonia, ethyl 
methyl ketone, butyric acid, acetic acid, and acetone in urine samples [16]. Therefore, the type of the sensors refers to these five types of VOCs. In acetone there is a propane element, so the MQ2 sensor is used. For ethyl methyl ketone and butyric acid in which there is butane, then the MQ4 and MQ7 sensors are involved. The MQ135 sensor is used to detect ammonia. A summary based on the datasheet on each sensor is listed in Table 1.

The sensor is a semiconductor type gas sensor. This gas sensor requires a certain temperature to work, so there is a heater in it. At high temperatures, an oxidation process occurs in the metal oxide $\left(\mathrm{SnO}_{2}\right)$ sensor material, and reduces it when the target gas makes contact with its surface. In clean air, the resistance of $\mathrm{SnO}_{2}$ is higher, and will decrease as the target gas concentration increases [22].

\subsection{Convolutional neural network}

CNN is one of the most common types of artificial neural networks used in computer vision and pattern recognition [23]. However, the utilization of $\mathrm{CNN}$ for 1-D signal processing applications requires precise 1-D to 2-D conversion. Several studies have tried to use deep CNN by converting 1-D signals to 2-D, among others for bearing fault diagnosis [24], and also ECG classification and arrhythmia detection $[25,26]$. The disadvantage of 2-D CNN is the high computational complexity. 1-D CNN has been developed to operate directly and more efficiently. Several studies have used it in signal processing applications, for early detection of arrhythmias [27,28], environmental noise classification [29], and detection of atrial fibrillation on ECG [30].

In general, $\mathrm{CNN}$ consists of a convolution layer, pooling layer and fully connected layer [31] with a rectified linear activation function [32]. The convolutional neural network architecture used in this proposed design consists of a convolution layer, max-pooling layer, flatten, fully connected layer. In this study, 1D-Convolution is used in the first convolution layer which is defined as

$$
y[i]=\sum_{j=0}^{M-1} h[j] \cdot x[i-j]
$$

Table 1. Sensor specifications

\begin{tabular}{|c|c|}
\hline Sensor & Response \\
\hline MQ2 & Methane, Propane \\
\hline MQ4 & Butane, Alcohol \\
\hline MQ7 & CO, Butane, Methane \\
\hline MQ135 & Ammonia, Benzene \\
\hline DHT11 & Temperature, Humidity \\
\hline
\end{tabular}

where $y$ is $i$-th output, $x$ is the input signal $N$ running from 0 to $N-1, h$ is a kernel of length $M$. The first layer uses 8 kernels, size $7 \times 1$, a stride $1 \times 1$, the activation function uses ReLu. The second layer uses a 1Dconvolution 8 kernel with a size of $7 \times 1$, a stride $1 \times$ 1 , the activation function uses ReLu. The ReLu function is defined as

$$
\operatorname{ReLu}=\left[\begin{array}{ll}
0, & x \leq 0 \\
x, & x>0
\end{array}\right]
$$

where $\mathrm{x}$ is the input value. The next process is $1 \mathrm{D}$ Max-pooling with a size of $2 \times 1$ and stride $2 \times 1$ used to reduce the size of the data on each sensor. The result of this Max-pooling process is $44 \times 8$. Then, the flatten process is carried out, resulting in a data size of $352 \times 1$. The first fully connected activation has 256 nodes. The second fully connected activation has 2 nodes which is the number of classes. Fig. 3 shows an illustration of CNN modeling in this study. The first fully connected activation function uses ReLu, while the second uses Softmax. The Softmax function is defined as

$$
\sigma(z)_{i}=\frac{e^{z_{i}}}{\sum_{j=1}^{K} e^{z_{j}}}
$$

where $\mathrm{z}$ is the input vector, $e^{z_{i}}$ is the standard exponential function for the input vector, $\mathrm{K}$ is the number of classes, $e^{z_{j}}$ is the standard exponential function for the output vector.

The learning process uses the Adaptive Moment Estimation (ADAM) optimizer which is a combination of the root mean square (RMS) propagation and momentum. The ADAM optimizer is expressed as

$$
\begin{gathered}
m_{t}=\beta_{1} m_{t-1}+\left(1-\beta_{1}\right) g_{t} \\
v_{t}=\beta_{2} v_{t-1}+\left(1-\beta_{2}\right)\left(g_{t}\right)^{2} \\
\omega_{t+1}=\omega_{t}-\alpha \frac{m_{t}}{\sqrt{v_{t}}+\varepsilon}
\end{gathered}
$$

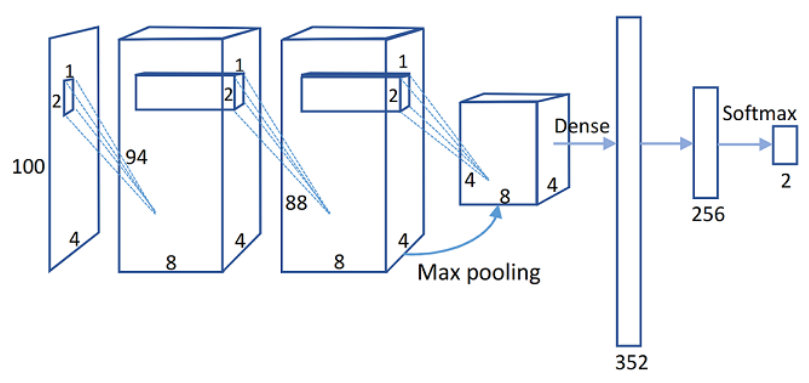

Figure. 3 CNN network model illustration 
where, $m_{t}$ is the exponentially weighted average of the previous gradient, $v_{t}$ is the exponentially weighted average of the squares of the previous gradient, $\beta_{1}, \beta_{2}$ is the adjustable hyper parameter, $g_{t}$ is the gradient at t. $\omega_{t+1}$ is the weight of the matrix to be updated, $\alpha$ is the learning rate, $\varepsilon$ is a very small value to avoid division of zero values.

Determination of the level of accuracy of the model uses loss function cross entropy. The cross entropy is defined as:

$$
L_{y}(y)=-\sum_{i=0}^{M} y_{i}^{\prime} \log \left(y_{i}\right)
$$

where $y$ is the probability distribution of a prediction, $y^{\prime}$ is the true probability distribution and $M$ is the number of classes.

In this study, the CNN model is run using the Tensorflow and Keras libraries, while Python is the programming language. This library is often used in pattern recognition and computer vision [33].

\section{Results and discussion}

Sensor data was recorded for 100 s at a sample rate of $1 \mathrm{~Hz}$ starting from the initial response of the gas sensor when the sample is inserted into the chamber until the response has stabilized. In one measurement, 100 points are obtained for a sensor, so for 4 sensors, $100 \times 4=400$ points are obtained. In data collection, interference occurs, both from inside and outside the system. Therefore, to avoid inaccurate data, signal processing is needed. The moving average method is used to reduce interference with the electronic nose signal. Sensor responses to urine samples are shown in Fig. 4.

In data processing, two treatments were carried out, the first using a total of 400 points. This data was used in the CNN method. Meanwhile, the second treatment was by taking the average value of each sensor. Therefore, we get 4 points for a sample. The data were used in the LVQ and MLP methods. Fig. 5 shows the pattern of sensor responses in diabetic and

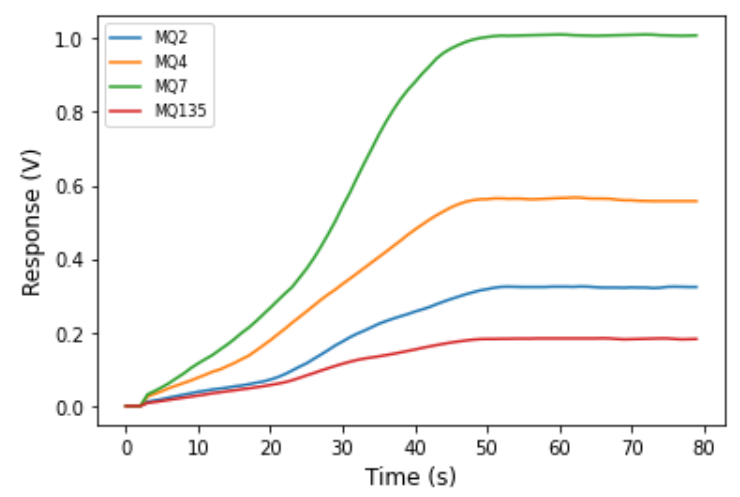

Figure. 4 Gas sensor response to urine sample

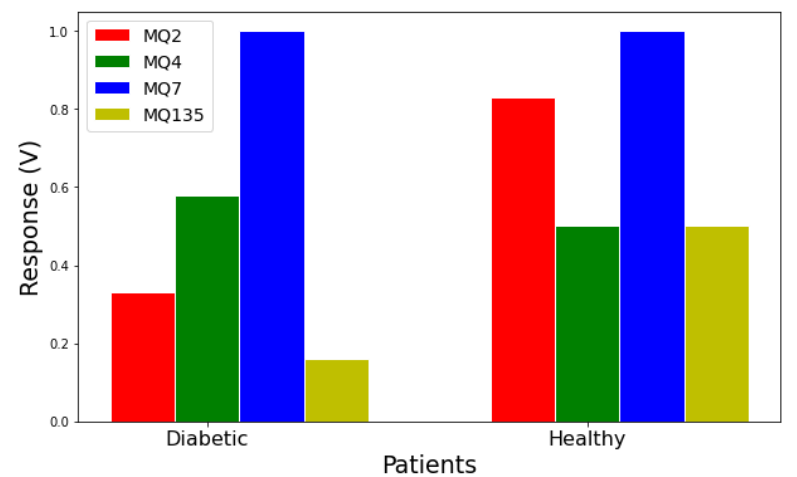

Figure. 5 Gas sensor response patterns to urine samples of diabetic and healthy patients

healthy patients. The composition of the data in this study is $70 \%$ for learning data and $30 \%$ for test data from a total of 50 data. The composition of the learning data consisted of 30 samples of diabetics and 5 healthy samples. The learning process in the LVQ method uses 256 neurons, learning rate of $0.2,0.4$, $0.1,0.09$, and 0.05 obtained an accuracy value of $60 \%$ with MSE $=0$. This value is the highest as given in Table 2. Likewise, for the MLP method using 256 neurons, TrainGDM as a training function, LearnGDM as a learning function, learning rates of 0.09 and 0.05 obtained an accuracy of $66.67 \%$. This value is the highest, as shown in Table 3 . In both methods, even though during the learning process a very low error value was obtained, the accuracy was not satisfactory.

Table 2. Testing the accuracy of the LVQ method

\begin{tabular}{|c|c|c|c|}
\hline LR & Epoch & MSE & Accuracy (\%) \\
\hline 0.05 & 59 & 0 & 60.0 \\
\hline 0.09 & 107 & 0 & 60.0 \\
\hline 0.1 & 37 & 0 & 60.0 \\
\hline 0.2 & 318 & 0 & 60.0 \\
\hline 0.4 & 282 & 0 & 60.0 \\
\hline 0.5 & 442 & 0 & 46.7 \\
\hline
\end{tabular}

Table 3. Testing the accuracy of the MLP method

\begin{tabular}{|c|c|c|c|c|}
\hline $\begin{array}{c}\text { Training } \\
\text { Function }\end{array}$ & LR & Epoch & MSE & $\begin{array}{c}\text { Accuracy } \\
(\boldsymbol{\%})\end{array}$ \\
\hline TrainLM & 0.05 & 9 & $1.13 \times 10^{-9}$ & 60.00 \\
\hline TrainLM & 0.09 & 9 & $7.73 \times 10^{-10}$ & 60.00 \\
\hline TrainLM & 0.1 & 9 & $6.65 \times 10^{-10}$ & 53.33 \\
\hline TrainGDM & 0.01 & 10000 & $5.95 \times 10^{-4}$ & 60.00 \\
\hline TrainGDM & 0.05 & 10000 & $8 \times 10^{-2}$ & 66.67 \\
\hline TrainGDM & 0.09 & 10000 & $6.7 \times 10^{-5}$ & 66.67 \\
\hline
\end{tabular}




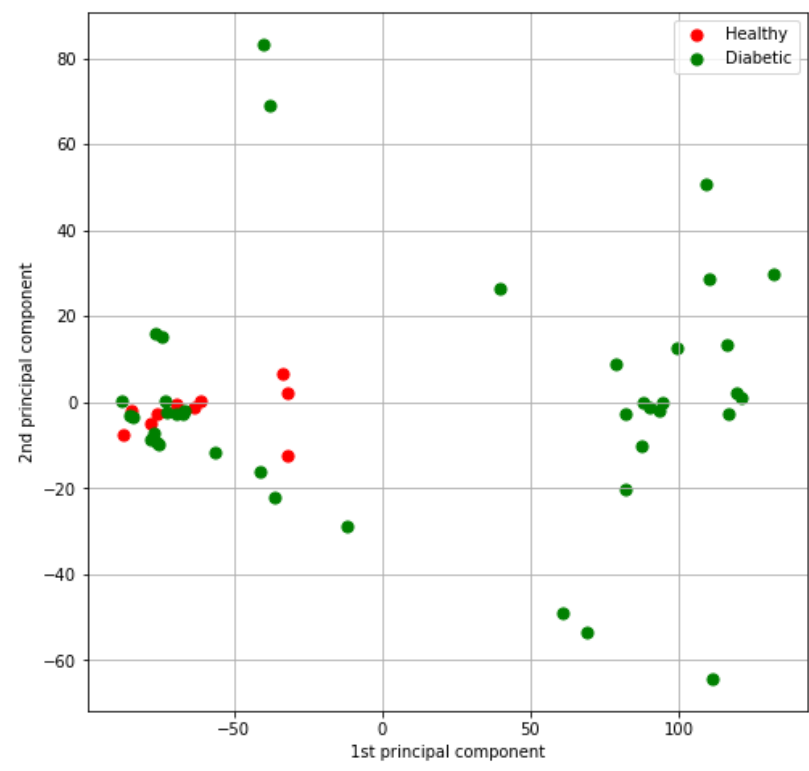

Figure. 6 PCA results without a scaler

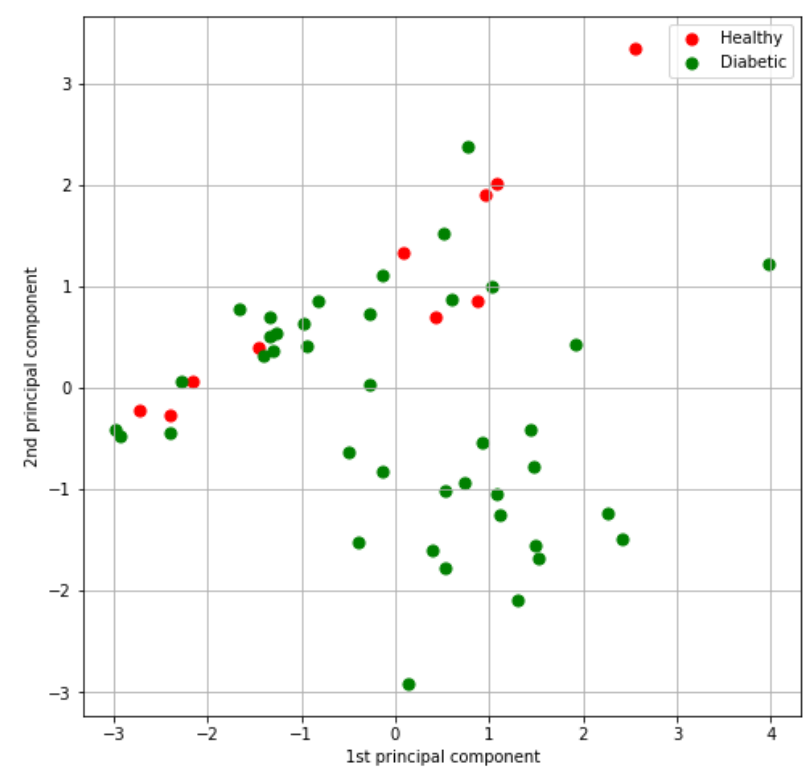

Figure. 7 PCA results with a scaler

The results of the evaluation of the two methods listed in Table 2 and Table 3 turned out to be unsatisfactory. Therefore, it is necessary to preprocess the data, because not all sensors have a significant contribution in the identification of diabetes. In this system, there are six sensors used as identification features, i.e., acetone (MQ2), ethyl methyl ketone (MQ4), butyric acid (MQ7), ammonia (MQ135), temperature, and humidity (DHT11). Sensor selection is needed to find the most dominant one in identification. To extract features, the principal component analysis (PCA) method is used. This method can reduce the size of the data without eliminating the characteristics of the data, by replacing the data with new variables that are linear with the original variables. The use of a scaler in PCA
Table 4. Results of the evaluation of the PCA-LVQ method

\begin{tabular}{|c|c|c|c|}
\hline LR & Epoch & MSE & $\begin{array}{c}\text { Accuracy } \\
(\boldsymbol{\%})\end{array}$ \\
\hline 0.05 & 64 & 0 & 73.3 \\
\hline 0.09 & 57 & 0 & 73.3 \\
\hline 0.1 & 36 & 0 & 80.0 \\
\hline 0.2 & 62 & 0 & 80.0 \\
\hline 0.4 & 40 & 0 & 53.3 \\
\hline 0.5 & 43 & 0 & 60.0 \\
\hline
\end{tabular}

Table 5. Results of the evaluation of the PCA-MLP

\begin{tabular}{|c|c|r|c|c|}
\hline $\begin{array}{c}\text { Training } \\
\text { Function }\end{array}$ & LR & Epoch & MSE & $\begin{array}{c}\text { Accuracy } \\
(\%)\end{array}$ \\
\hline TrainLM & 0.01 & 9 & $5.7 \times 10^{-10}$ & 80.00 \\
\hline TrainLM & 0.05 & 7 & $2.4 \times 10^{-9}$ & 80.00 \\
\hline TrainLM & 0.09 & 8 & $4.3 \times 10^{-9}$ & 80.00 \\
\hline TrainGDM & 0.01 & 10000 & $4.67 \times 10^{-5}$ & 86.67 \\
\hline TrainGDM & 0.05 & 10000 & $8.8 \times 10^{-6}$ & 80.00 \\
\hline TrainGDM & 0.09 & 10000 & $4.5 \times 10^{-6}$ & 80.00 \\
\hline
\end{tabular}

Table 6. Summary of the first model CNN architecture

\begin{tabular}{|c|l|c|c|c|}
\hline Layer & \multicolumn{1}{|c|}{ Type } & $\begin{array}{c}\text { Output } \\
\text { Shape }\end{array}$ & $\begin{array}{c}\text { Kernel } \\
\text { size }\end{array}$ & Stride \\
\hline $0-1$ & Convolution 1D & 92,16 & $9 \times 1$ & $1 \times 1$ \\
\hline $1-2$ & Max-pooling 1D & 46,16 & $2 \times 1$ & $2 \times 1$ \\
\hline $2-3$ & Convolution 1D & 38,16 & $9 \times 1$ & $1 \times 1$ \\
\hline $3-4$ & Max-pooling 1D & 19,16 & $2 \times 1$ & $2 \times 1$ \\
\hline $4-5$ & Dropout & 19,16 & - & - \\
\hline $5-6$ & Flatten & 304 & - & - \\
\hline $6-7$ & Fully-connected & 256 & - & - \\
\hline $7-8$ & Fully-connected & 2 & - & - \\
\hline
\end{tabular}

makes for better results. This can be seen in Fig. 6 that shows data accumulation of diabetic and healthy patients. Meanwhile, Fig. 7 shows that there is only a slight accumulation of data between healthy and diabetic patients. The standard scaler is defined as

$$
z=\frac{x-\mu}{\sigma}
$$

where $\mathrm{x}$ is the input, $\mu$ is the mean of the input and $\sigma$ is the standard deviation.

The results of the PCA-LVQ method evaluation with 3 principal components as input are given in Table 4 . The highest accuracy is obtained at $80 \%$. This result is better than without PCA. The PCAMLP method evaluation with 3 principal components as input is listed in Table 5. The evaluation results 
obtained the highest accuracy of $86.6 \%$. The next method uses the first $\mathrm{CNN}$ model, which consists of a convolutional layer, max-pooling, convolutional layer, Max-pooling, drop out, flatten, and fully connected activation. A summary of the architecture of the first model is listed in Table 6 .

The training loss curve is one of the most important evaluations in measuring the success of a pattern recognition algorithm. Fig. 8 shows each decreasing training loss curve with increasing epoch.

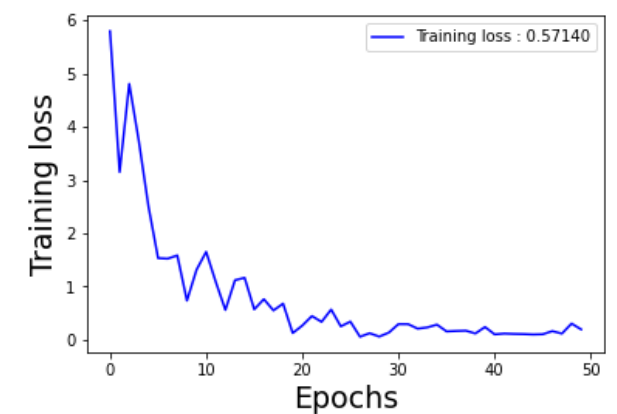

(a)

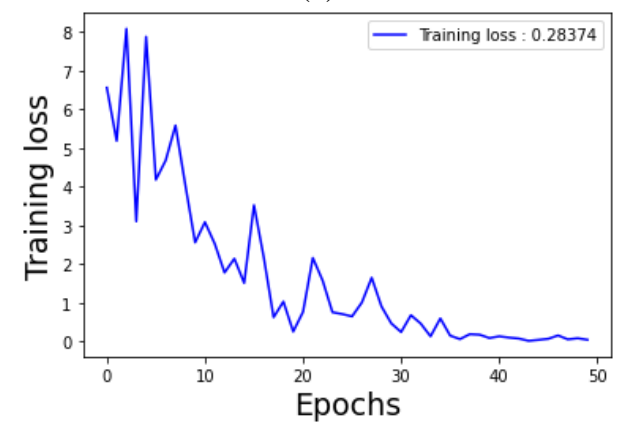

(b)

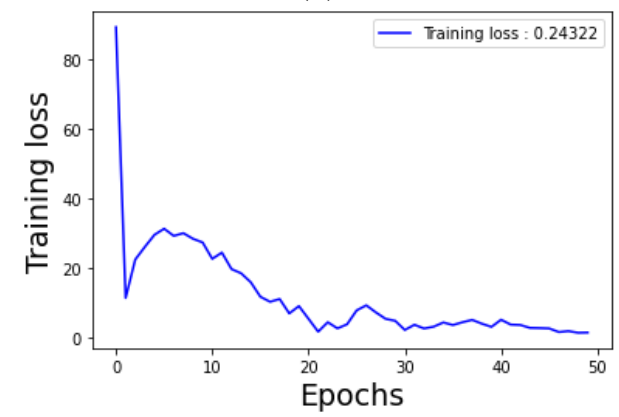

(c)

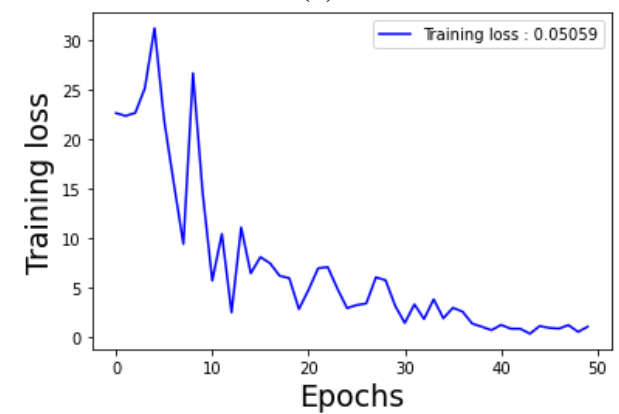

(d)

Figure. $8 \mathrm{CNN}$ training losses for accuracy: (a) 80\%, (b) $87 \%$, (c) $93 \%$, and (d) $100 \%$
The best weight and bias values were used to identify 15 data. The number of neurons in fully connected activation in the training process is 256 . When the training losses are $0.57,0.28,0.24$, and 0.05 with 50 epochs, each produces an accuracy of $80 \%, 87 \%$, $93 \%$, and $100 \%$, respectively.

The evaluation of the first CNN model was carried out with several kernels consisting of 8,16 , 32 , and 64 with kernel sizes including $3 \times 1,5 \times 1,7 \times 1$, and $9 \times 1$, the highest accuracy was obtained at $100 \%$. Table 7 shows the average results of 3 tests. The best accuracy results for the first model were obtained with 16 kernel sizes, which were $9 \times 1$ with an average of $98 \%$. The performance of the first model has been evaluated. Furthermore, the evaluation of the second model was carried out with the architecture as shown in Table 8. The process of learning and testing was treated the same as the first model. The best accuracy results for the second model were obtained with 8 kernels, size $7 \times 1$, as shown in Table 7 with an average of $100 \%$. Both CNN models produced the highest accuracy value of $100 \%$. Both models have good performance in identification as shown in Table 7.

The second model with 8 kernels, size $7 \times 1$ has a better average accuracy value than the first model. The results of the identification of the second CNN model with 8 kernels, size $7 \times 1$ and accompanied by clinical testing are shown in Table 9. If the clinical results show more than $180 \mathrm{mg} / \mathrm{dL}$ of blood sugar, it indicates a person has a high risk of diabetes [34].

Table 7. Testing the first and second CNN models

\begin{tabular}{|c|c|c|c|}
\hline \multirow{2}{*}{$\begin{array}{c}\text { Number } \\
\text { of } \\
\text { Kernel }\end{array}$} & \multirow{2}{*}{$\begin{array}{c}\text { Kernel } \\
\text { Size }\end{array}$} & \multicolumn{2}{|c|}{ Accuracy (\%) } \\
\cline { 3 - 4 } & & \multicolumn{2}{|c|}{ Models } \\
\hline 8 & $3 \times 1$ & 78 & Second \\
\hline 8 & $5 \times 1$ & 91 & 95 \\
\hline 8 & $7 \times 1$ & 75 & 85 \\
\hline 8 & $9 \times 1$ & 93 & 100 \\
\hline 16 & $3 \times 1$ & 76 & 87 \\
\hline 16 & $5 \times 1$ & 84 & 93 \\
\hline 16 & $7 \times 1$ & 87 & 93 \\
\hline 16 & $9 \times 1$ & 98 & 95 \\
\hline 32 & $3 \times 1$ & 87 & 87 \\
\hline 32 & $5 \times 1$ & 89 & 89 \\
\hline 32 & $7 \times 1$ & 91 & 82 \\
\hline 32 & $9 \times 1$ & 89 & 82 \\
\hline 64 & $3 \times 1$ & 87 & 93 \\
\hline 64 & $5 \times 1$ & 82 & 84 \\
\hline 64 & $7 \times 1$ & 91 & 80 \\
\hline 64 & $9 \times 1$ & 91 & 100 \\
\hline & & & 91 \\
\hline
\end{tabular}


Table 8. Summary of the second model CNN architecture

\begin{tabular}{|c|l|c|c|c|}
\hline Layer & \multicolumn{1}{|c|}{ Type } & $\begin{array}{c}\text { Output } \\
\text { Shape }\end{array}$ & $\begin{array}{c}\text { Kernel } \\
\text { size }\end{array}$ & Stride \\
\hline $0-1$ & Convolution $1 D$ & 94,8 & $7 \times 1$ & $1 \times 1$ \\
\hline $1-2$ & Convolution $1 D$ & 88,8 & $7 \times 1$ & $1 \times 1$ \\
\hline $2-3$ & Dropout & 88,8 & - & - \\
\hline $3-4$ & Max-pooling 1D & 44,8 & $2 \times 1$ & $2 \times 1$ \\
\hline $4-5$ & Flatten & 352 & - & - \\
\hline $5-6$ & Fully-connected & 256 & - & - \\
\hline $6-7$ & Fully-connected & 2 & - & - \\
\hline
\end{tabular}

The confusion matrix is one way to evaluate the identification method. This is usually described in a comparison between the actual condition with the prediction. In this study, the confusion matrix was used to evaluate the second CNN model method in identifying diabetic and healthy patients. Fig. 9 shows that there are 10 diabetic patients according to the actual condition and 5 healthy according to the actual condition. For further analysis, the CNN identification results are divided into True Positive Rate (TPR), False Positive Rate (FPR), True Negative Rate (TNR), and False Negative Rate (FNR) which are defined as follows:

$$
\begin{aligned}
& T P R=\frac{T P}{T P+F N} \\
& F P R=\frac{F P}{F P+T N} \\
& T N R=\frac{T N}{T N+F P} \\
& F N R=\frac{F N}{F N+T P}
\end{aligned}
$$

Table 9. Identification of diabetic and healthy patients

\begin{tabular}{|c|c|c|c|}
\hline No & $\begin{array}{c}\text { Clinical Test } \\
(\mathbf{m g} / \mathbf{d L})\end{array}$ & Patient & Identification \\
\hline 1 & 93 & Healthy & Healthy \\
\hline 2 & 65 & Healthy & Healthy \\
\hline 3 & 63 & Healthy & Healthy \\
\hline 4 & 58 & Healthy & Healthy \\
\hline 5 & 54 & Healthy & Healthy \\
\hline 6 & 290 & Diabetic & Diabetic \\
\hline 7 & 193 & Diabetic & Diabetic \\
\hline 8 & 235 & Diabetic & Diabetic \\
\hline 9 & 315 & Diabetic & Diabetic \\
\hline 10 & 311 & Diabetic & Diabetic \\
\hline 11 & 317 & Diabetic & Diabetic \\
\hline 12 & 198 & Diabetic & Diabetic \\
\hline 13 & 286 & Diabetic & Diabetic \\
\hline 14 & 310 & Diabetic & Diabetic \\
\hline 15 & 205 & Diabetic & Diabetic \\
\hline
\end{tabular}

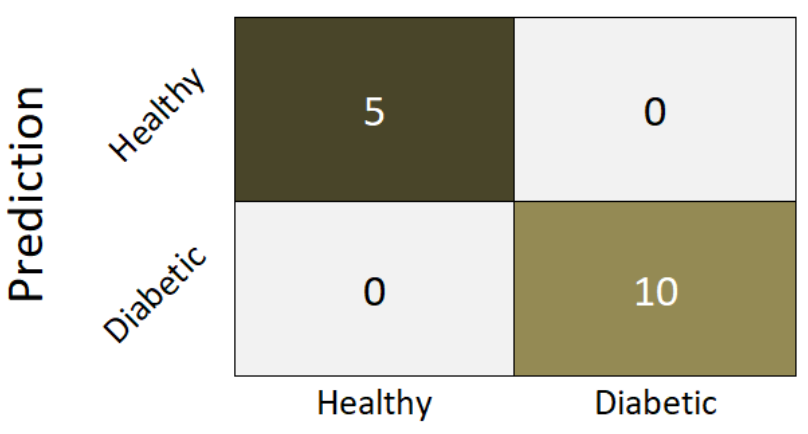

Actual

Figure. 9 The results of the confusion matrix

Table 10. Measurement of the performance of the second model CNN

\begin{tabular}{|c|c|c|c|c|c|c|c|}
\hline TP & FP & TN & FN & TPR & FPR & TNR & FNR \\
\hline 10 & 5 & 0 & 0 & 1 & 1 & 0 & 0 \\
\hline
\end{tabular}

Table 11. Comparison of the eight pattern recognition methods

\begin{tabular}{|c|c|c|c|}
\hline Methods & $\begin{array}{c}\text { Accuracy } \\
(\boldsymbol{\%})\end{array}$ & $\begin{array}{c}\text { Precision } \\
(\boldsymbol{\%})\end{array}$ & $\begin{array}{c}\text { Sensitivity } \\
(\boldsymbol{\%})\end{array}$ \\
\hline LVQ & 60 & 70 & 70 \\
\hline MLP & 66.7 & 100 & 66.7 \\
\hline Naïve Bayes & 66.7 & 100 & 66.7 \\
\hline k-NN & 86.7 & 100 & 86 \\
\hline SVM & 80 & 100 & 80 \\
\hline PCA-LR & 86.7 & 100 & 86 \\
\hline PCA-LVQ & 80 & 100 & 80 \\
\hline PCA-MLP & 86.7 & 100 & 86 \\
\hline CNN & 100 & 100 & 100 \\
\hline
\end{tabular}

TPR is used to measure the proportion of positives that are identified as true or also called sensitivity. TPR value of 1 indicates that the evaluation of the CNN method in identifying is very good as listed in Table 10.

Overall comparison of LVQ, MLP, Naïve Bayes methods with Bernoulli function, $\mathrm{k}-\mathrm{NN}$ with $\mathrm{k}=3$, SVM with radial basis function kernel, PCA-LR, PCA-LVQ, PCA-MLP and CNN in identifying is given in Table 11. The CNN method has a better accuracy rate than other methods.

Several previous studies on electronic nose to classify diabetes through urine vapor have been carried out. The classification methods used include Cluster Analysis and PCA with an accuracy of $99.5 \%$ [16], the PCA-LR method with an accuracy of $95 \%$ [35], and the enhanced adaptive-genetic algorithmMLP (EAGA-MLP) method that can diagnose diabetes $94.7 \%$ [36]. Similar research has also been carried out, but through human breathing. The method was by optimizing deep neural network that can identify diabetes $96.29 \%$ [18]. This shows that the classification method for detecting diabetes 
through urine vapor samples using gas sensors and the CNN method has higher accuracy.

\section{Conclusion}

In this study, an electronic nose system has been developed using metal oxide sensors array and a CNN pattern recognition algorithm. LVQ, MLP, kNN, SVM, PCA-LR, PCA-LVQ and PCA-MLP are as comparison methods. This system is used to identify diabetes through urine. The learning stage is to get the best model, weights, and bias for each method. From the eight methods, it was found that $\mathrm{CNN}$ has a high level of accuracy in identifying two classes (healthy and diabetic). There are two CNN models applied in the identification. The evaluation results obtained that the model with 8 kernels, size $7 \times 1$ has a higher accuracy rate of $100 \%$. The future work will implement the electronic nose into a more compact and portable embedded system for diabetes detection. In addition, this system is expected to be an alternative method that is cheaper, easier, more convenient, and avoids the risk of infection.

\section{Conflicts of Interest}

The authors declare no conflict of interest.

\section{Author Contributions}

In this research article author contribution are as follows: "Conceptualization and Methodology, Misbah, Muhammad Rivai, and Fredy Kurniawan; software, Dava Aulia; data curation, Zainul Muchidin, and Misbah; Writing-original draft preparation, Misbah; writing-review and editing by Misbah, and Muhammad Rivai; supervision, Muhammad Rivai and Fredy Kurniawan".

\section{Acknowledgments}

We would like to thank Kementerian Riset dan Teknologi/Badan Riset dan Inovasi Nasional Republik Indonesia for financial aid support.

\section{References}

[1] E. W. Gregg, Y. J. Cheng, M. Srinivasan, J. Lin, L. S. Geiss, A. L. Albright, and G. Imperatore, "Trends in cause-specific mortality among adults with and without diagnosed diabetes in the USA: an epidemiological analysis of linked national survey and vital statistics data", Lancet, Vol. 391, No. 10138, pp. 2430-2440, 2018.

[2] N. R. Burrows, Y. F. Li, E. W. Gregg, and L. S. Geiss, "Declining rates of hospitalization for selected cardiovascular disease conditions among adults aged 35 years with diagnosed diabetes, U.S., 1998-2014”, Diabetes Care, Vol. 41, No. 2, pp. 293-302, 2018.

[3] M. Markoulli, J. Flanagan, S. S. Tummanapalli, J. Wu, and M. Willcox, "The impact of diabetes on corneal nerve morphology and ocular surface integrity", The Ocular Surface, Vol. 16, No. 1, pp. 45-57, 2018.

[4] D. N. Koye, D. J. Magliano, R. G. Nelson, and M. E. Pavkov, "The global epidemiology of diabetes and kidney disease", Advances in Chronic Kidney Disease, Vol. 25, No. 2, pp. 121-132, 2018.

[5] D. R. Rojas, R. Kuner, and N. Agarwal, "Metabolomic signature of type 1 diabetesinduced sensory loss and nerve damage in diabetic neuropathy", Journal of Molecular Medicine, Vol. 97, No. 6, pp. 845-854, 2019.

[6] G. Balzano, P. Maffi, R. Nano, A. Mercalli, R. Melzi, F. Aleotti, F. D. Cobelli, P. Magistretti, and M. Scavini, "Diabetes-free survival after extended distal pancreatectomy and islet auto transplantation for benign or borderline/malignant lesions of the pancreas", American Journal of Transplantation, Vol. 19, No. 3, pp. 920-928, 2019.

[7] R. Gundamaraju and R. Vemuri, "Pathophysiology of Greedy Colon and Diabetes: Role of Atropine in worsening of Diabetes", Euroasian Journal of HepatoGastroenterology, Vol. 4, No. 1, pp. 51-54, 2014.

[8] N. Zhang, F. Geng, Z. H. Hue, B. Liu, Y. Q. Wang, J. C. Liu, Y. H. Qi, and L. J. Li, "Preliminary study of urine metabolism in type two diabetic patients based on GC-MS", American Journal of Translational Research, Vol. 8, No. 7, pp. 2889-2896, 2016.

[9] M. A. Hasan, R. Sarno, and S. I. Sabilla, "Optimizing machine learning parameters for classifying the sweetness of pineapple aroma using electronic nose", International Journal of Intelligent Engineering Systems, Vol. 13, No. 5, pp. 122-132, 2020.

[10] Misbah, M. Rivai, and F. Kurniawan, "Quartz crystal microbalance based electronic nose system implemented on Field Programmable Gate Array", Telkomnika (Telecommunication Comput. Electron. Control, Vol. 17, No. 1, pp. 370-376, 2019.

[11] M. Rivai, Misbah, M. Attamimi, M. H. Firdaus, Tasripan, and Tukadi, "Fish Quality Recognition using Electrochemical Gas Sensor Array and Neural Network", In: Proc. of International Conf. on Computer Engineering, 
Network and Intelligent Multimedia, pp. 28-32, 2019.

[12] S. Wakhid, R. Sarno, S. I. Sabilla, and D. B. Maghfira, "Detection and classification of indonesian civet and non-civet coffee based on statistical analysis comparison using E-Nose", International Journal of Intelligent Engineering Systems, Vol. 13, No. 4, pp. 56-65, 2020.

[13] H. A. Sujono, M. Rivai, and M. Amin, "Asthma Identification Using Gas Sensors and Support Vector Machine", Telkomnika, Vol. 16, No. 4, pp. 1468-1480, 2018.

[14] L. Singh, R. Singh, B. Zhang, S. Cheng, B. K. Kaushik, and S. Kumar, "LSPR Based Uric Acid Sensor Using Graphene Oxide and Gold Nanoparticles Functionalized Tapered Fiber", Optical Fiber Technology, Vol. 53, pp. 1-8, 2019.

[15] N. E. Azmi, A. H. A. Rashid, J. Abdullah, N. A. Yusof, and H. Sidek, "Fluorescence biosensor based on encapsulated quantum dots/enzymes/sol-gel for non-invasive detection of uric acid", Journal of Luminescene, Vol. 202, pp. 309-315, 2018.

[16] T. Seesaard, C. Sriphrapradang, T. Kitiyakara, and T. Kerdcharoen, "Self-screening for diabetes by sniffing urine samples based on a hand-held electronic nose", In: Proc. of International Conf. on Biomedical Engineering, pp. 1-4, 2016.

[17] Q. Dou, D. Hu, H. Gao, Y. Zhang, A. K. Yetisen, H. Butt, J. Wang, G. Nie, and Q. Dai, "High performance boronic acid-containing hydrogel for biocompatible continuous glucose monitoring", Royal Society of Chemistry, Vol. 7, No. 66, pp. 41384-41390, 2017.

[18] R. Sarno, S. I. Sabilla, and D. Rahmanwijaya, "Electronic Nose for Detecting Multilevel Diabetes using Optimized Deep Neural Network", Engineering Letters, Vol. 28, No. 1, pp. 31-42, 2020.

[19] A. Hamza and H. Moetque, "Diabetes Disease Diagnosis Method based on Feature Extraction using K-SVM", International Journal Advanced Computer Science and Applications, Vol. 8, No. 1, pp. 236-244, 2017.

[20] N. Pradhan, G. Rani, V. S. Dhaka, and R. C. Poonia, "Diabetes prediction using artificial neural network", Deep Learn. Tech. Biomed. Heal. Informatics, Vol. 121, pp. 327-339, 2020.

[21] P. Peng, X. Zhao, X. Pan, and W. Ye, "Gas Classification using Deep Convolutional Neural Networks", Sensors (Switzerland), Vol. 18, No. 1, p. 157, 2018
[22] X. Zhou, Y. Wang, J. Wang, Z. Xie, X Wu, N. Han, and Y. Chen, "Amplifying the Signal of Metal Oxide Gas Sensors for Low Concentration Gas Detection", IEEE Sensors Journal, Vol. 17, No. 9, pp. 2841-2847, 2017.

[23] D. X. Zhou, "Universality of deep convolutional neural networks", Applied and Computational Harmonic Analysis, Vol. 48, No. 2, pp. 787-794, 2020.

[24] Z. Wei, P. Gaoliang, and L. Chuanhao, "Bearings Fault Diagnosis Based on Convolutional Neural Networks with 2-D Representation of Vibration Signals as Input", In: Proc. of International Conf. on Mechatronics and Mechanical Engineering, pp. 1-5, 2016.

[25] M. Zihlmann, D. Perekrestenko, and M. Tschannen, "Convolutional recurrent neural networks for electrocardiogram classification", In: Proc. of International Conf. on Computing in Cardiology (CinC), pp. 1-4, 2017.

[26] J. T. Ruiz, J. B. Blázquez, and J. Ramón. "Arrhythmia Detection Using Convolutional Neural Models", In: Proc. of International Conf. on Distributed Computing and Artificial Intelligence, pp. 120-127, 2018.

[27] S. Kiranyaz, T. Ince, and M. Gabbouj, "Personalized Monitoring and Advance Warning System for Cardiac Arrhythmias", Scientific Reports, Nature, Vol. 7, No. 1, 2017.

[28] U. R. Acharya, H. Fujita, O. S. Lih, Y. Hagiwara, J. H. Tan, and M. Adam, "Automated detection of arrhythmias using different intervals of tachycardia ECG segments with convolutional neural network", Information Sciences, Vol. 405, pp. 81-90, 2017.

[29] S. Abdoli, P. Cardinal, and A. L. Koerich, "Endto-end environmental sound classification using a 1D convolutional neural network", Expert Systems with Applications, Vol. 136, pp. 252263, 2019.

[30] C. H. Hsieh, Y. S. Li, B. J. Hwang, and C. H. Hsiao, "Detection of atrial fibrillation using 1D convolutional neural network", Sensors, Vol. 20, No. 7, 2020.

[31] J. Gu, Z. Wang, J. Kuen, L. Ma, A. Shahroudy, B. Shuai, T. Liu, X. Wang, G. Wang, J. Cai, and T. Chen, "Recent Advances in Convolutional Neural Networks", Pattern Recognition, Vol. 77, pp. 354-377, 2018.

[32] X. Jiang, Y. Pang, X. Li, J. Pan, and Y. Xie, "Deep neural networks with Elastic Rectified Linear Units for object recognition", Neurocomputing, Vol. 275, pp. 1132-1139, 2018.

[33] B. J. Erickson, P. Korfiatis, Z. Akkus, T. Kline, and K. Philbrick, "Toolkits and Libraries for 
Deep Learning", Journal of Digital Imaging, Vol. 30, No. 4, pp. 400-405, 2017.

[34] G. Giath, "Management of an emergency tooth extraction in diabetic patients on the dental chair", The Saudi Journal, Vol. 32, No. 1, pp. 16, 2020.

[35] S. Esfahani, A. Wicaksono, E. Mozdiak, and R. P. Arasaradnam, "Non-Invasive Diagnosis of Diabetes by Volatile Organic Compounds in Urine Using FAIMS and Fox4000 electronic nose", Biosensors, Vol. 8, No. 4, pp. 121-134, 2018.

[36] S. Mishra, H. K. Tripathy, P. K. Mallick, A. K. Bhoi, and P. Barsocchi, "EAGA-MLP-An Enhanced and Adaptive Hybrid Classification Model for Diabetes Diagnosis", Sensors, Vol. 20, No. 14, p. 4036, 2020. 